\title{
On the multiwavelength emission from gamma ray burst afterglows
}

\author{
M. Petropoulou and A. Mastichiadis
}

Department of Physics, University of Athens, Panepistimiopolis, GR 15783 Zografou, Greece

e-mail: amastich@phys.uoa.gr

Received 23 July 2009 / Accepted 21 August 2009

ABSTRACT

\begin{abstract}
Aims. Drawing an analogy with active galactic nuclei, we investigate the one-zone synchrotron self-compton (SSC) model of gamma ray bursts (GRB) afterglows in the presence of electron injection and cooling both by synchrotron and SSC losses.

Methods. We solve the spatially averaged kinetic equations which describe the simultaneous evolution of particles and photons, obtaining the multi-wavelength spectrum as a function of time. We back up our numerical calculations with analytical solutions of the equations using various profiles of the magnetic field evolution under certain simplifying assumptions.

Results. We apply the model to the afterglow evolution of GRBs in a uniform density environment and examine the impact various parameters have on the multiwavelength spectra. We find that in cases where the electron injection and/or the ambient density is high, the losses are dominated by SSC and the solutions depart significantly from the ones derived in the synchrotron standard cases.
\end{abstract}

Key words. gamma rays: bursts - acceleration of particles - radiation mechanisms: non-thermal

\section{Introduction}

Gamma ray burst (GRB) afterglows are thought to be produced in the relativistic blast waves (RBW) associated with the initial GRB explosion. According to the standard model - for a review see Piran (2005), the RBW expands in the circumburst material and, after sweeping some critical amount of mass, it starts to decelerate. At the same time electrons are postulated to energize at the shock front and radiate through synchrotron (Sari et al. 1998; Dermer \& Chiang 1998) or synchrotron and synchrotron self-compton (SSC) (Chiang \& Dermer 1999; Fan et al. 2008; Waxman 1997; Panaitescu \& Kumar 2000), thus producing the afterglow emission.

The above picture carries certain analogies to the radiation models put forward during the last decade to explain the multiwavelength (MW) observations of blazar emission, and, especially, to the "one-zone" SSC models (for recent reviews see Böttcher 2007; and Mastichiadis 2009). These models are based on solving an equation for the electron distribution function including synchrotron and SSC losses and, at the same time, calculating the radiated photon spectrum. This approach allows for time dependency to be taken explicitly into account (and thus it can address, for example, blazar flaring), it can treat the non-linear cooling associated with SSC and, moreover, it is self-consistent.

Motivated by these developments, we have applied the above technique to GRB afterglows. The aim of the present paper is not to fit spectra or lightcurves, but to focus on the impact the various parameters customarily used by researchers in the GRB field have on the MW spectra. We also present analytical solutions of the electron equation, under certain simplifying assumptions, for a power-law electron injection suffering synchrotron losses. This, allowed us to derive analytical results of the slopes of the flux time profiles and, at the same time, test the numerical code. In the present paper we restrict our analysis to the uniform density case.

The present paper has certain improvements over past efforts in the field (Chiang \& Dermer 1999). First, in the analytical solutions, we consider both standard and non-standard magnetic field evolution. We also use the full emissivity for synchrotron radiation instead of the $\delta$-function approximation. As far as the numerical part is concerned, we show that the inclusion of SSC both as electron energy loss mechanism and radiation process can bring, under certain circumstances, significant departures from the standard solutions which include only synchrotron radiation as an energy loss mechanism. Finally, we have included for the first time to the best of our knowledge, $\gamma \gamma$ absorption both as a $\gamma$-ray attenuation and particle reinjection mechanism.

The paper is structured as follows. In Sect. 2 we review the basic hydrodynamics and radiative concepts of the GRB afterglow adopted in the extensive literature on the subject. We also draw an analogy to the blazar case. In Sect. 3 we present the analytical results for various profiles of the magnetic field. In Sect. 4 we present the numerical code and the tests we have performed to check its validity. In Sect. 5 we show various numerical results and we conclude in Sect. 6 with a summary and a discussion.

\section{Physics of the relativistic blast wave}

\subsection{Hydrodynamics}

We assume a shell of material with initial mass $M_{0}$ moving with initial bulk Lorentz factor $\Gamma_{0}$. This will sweep ambient matter and will start decelerating with a rate determined by energy and momentum conservation. The resulting blast wave is modeled as having a cross sectional area, $A(r)$, that depends on the distance (measured in the framework of the explosion). In our work we consider a spherical blast wave with $A(r)=4 \pi r^{2}$ sweeping a constant density ambient matter. We also assume that the bulk kinetic energy which is converted to internal energy is not radiated away, contributing to the inertia of the blast wave (non-radiative limit). In this case the deceleration of the blast wave is determined by a pair of ordinary differential equations (Blandford \& McKee 1976):

$\frac{\mathrm{d} \Gamma}{\mathrm{d} r}=-\frac{A(r) \rho(r)\left(\Gamma^{2}(r)-1\right)}{M(r)}$ 
$\frac{\mathrm{d} M}{\mathrm{~d} r}=\rho(r) A(r) \Gamma(r)$

where $\Gamma=\Gamma(r)$ is the bulk Lorentz factor of the material, $M=$ $M(r)$ is the total mass including internal kinetic energy and $\rho=$ $\rho(r)$ is the mass density of the ambient matter. Note that Eq. (2) implies a non-radiative blastwave evolution. We will adopt this assumption throughout the paper.

It can be shown (Blandford \& McKee 1976) that the above system has an analytic solution given by:

$\Gamma(r)=\frac{\lambda\left(x^{3}-1\right)+\Gamma_{0}}{\left(1+2 \Gamma_{0} \lambda\left(x^{3}-1\right)+\lambda^{2}\left(x^{3}-1\right)^{2}\right)^{1 / 2}}$,

where $\lambda=\frac{4 \pi \rho_{0} r_{0}{ }^{3}}{3 M_{0}}, x=\frac{r}{r_{0}}$ and $M_{0}=\frac{E_{0}}{\Gamma_{0} c^{2}}$. Here $r_{0}$ is the initial radius of the blast wave and $\rho_{0}$ is the mass density of the uniform ambient matter. The evolution of the bulk Lorentz factor can be separated into three regimes. The first regime corresponds to the initial period of free expansion of the blast wave, during which $\Gamma(r) \approx \Gamma_{0}$. The decelerating phase follows, where the bulk Lorentz factor can be modeled as

$\Gamma(r)=\frac{\Gamma_{0}}{2}\left(\frac{r}{R_{\mathrm{d}}}\right)^{-3 / 2}=\sqrt{\frac{\Gamma_{0}}{4 \lambda}}\left(\frac{r_{0}}{r}\right)^{3 / 2}=\tilde{\Gamma} r^{-3 / 2}$

where

$\tilde{\Gamma}=\frac{\Gamma_{0}}{2} R_{\mathrm{d}}^{3 / 2}$

and

$R_{\mathrm{d}}=\left(\frac{3 E_{0}}{4 \pi n m_{\mathrm{p}} c^{2} \Gamma_{0}^{2}}\right)^{1 / 3}$

is the deceleration radius of the blast wave (Rees \& Mészáros 1992). This power-law $r^{-3 / 2}$ dependence of the bulk Lorentz factor is often quoted for a non-radiative blast wave decelerating in a uniform medium. During the third and final regime the blast wave is practically non-relativistic.

Energy conservation gives the rate of accreted kinetic energy in the lab frame:

$\frac{\mathrm{d} E}{\mathrm{~d} t}=c^{3} A(r) \rho(r) \beta\left(\Gamma^{2}(r)-\Gamma(r)\right)$.

This expression follows from the equation of motion (Eq. (1)) and is applied regardless of whether the blast wave is in the radiative or adiabatic regime. As the rate of energy accreted is a Lorentz invariant, expression (7) holds also in the comoving frame of the blast wave.

\subsection{Radiation}

The RBW not only sweeps matter and decelerates, as was discussed above, but it is assumed to be able to energize particles as well. While there are no detailed models as yet to explain the way particles achieve high energies, it is assumed that an ad-hoc fraction $\epsilon_{\mathrm{e}}$ of the accreted kinetic energy is injected into nonthermal electrons with a power-law form. A second assumption concerns the lower and upper cutoffs of the electron distribution $\gamma_{\min }$ and $\gamma_{\max }$. Since the normalization $\tilde{q}_{0}$ of the electron injection is set by the relation

$\int_{\gamma_{\min }}^{\gamma_{\max }} \tilde{q}_{0} \gamma^{-p}(\gamma-1) m_{\mathrm{e}} c^{2} \mathrm{~d} \gamma=\epsilon_{\mathrm{e}} \frac{\mathrm{d} E}{\mathrm{~d} t}$, where $\gamma$ is the Lorentz factor of the electrons and $p$ is the electron injection spectral index, it is evident that for $p>2$ (as is typically assumed), only the lower limit $\gamma_{\min }$ will play a role in determining the integral in Eq. (8) and thus $\tilde{q}_{0}$. Therefore the choice of $\gamma_{\min }$ has important consequences for the results. This can be at least of the order of $\Gamma$ in the comoving frame (Mastichiadis \& Kazanas 2009) but in the case where the electrons are in equipartition with protons it can be a factor of $\left(m_{\mathrm{p}} / m_{\mathrm{e}}\right)$ higher, i.e. $\gamma_{\min }=\left(m_{\mathrm{p}} / m_{\mathrm{e}}\right) \Gamma($ Katz \& Piran 1997; Panaitescu \& Mészáros 1998).

Since the energetic electrons will emit synchrotron radiation, a prescription for the magnetic field is also required. This again is a source of major uncertainty. The usual assumption is that the magnetic field is in some type of equipartition with the particles; this implies that the magnetic energy density takes a fraction $\epsilon_{\mathrm{B}}$ of the mass accumulated in the RBW, so the magnetic field is given by the relation

$B=\left(32 \pi n m_{\mathrm{p}} \epsilon_{\mathrm{B}} c^{2}\right)^{1 / 2} \Gamma$.

However one can consider different types of behavior, like $B \propto r^{-1}$ (Vlahakis \& Königl 2003).

Depending on the value of the magnetic field, the electrons can cool (i.e. radiate all their energy) in a dynamical timescale or remain uncooled, (i.e. keep their energy). These two cases have been called "fast" and "slow" cooling (Sari et al. 1998). From the standard solutions of the electron kinetic equations (Kardashev 1962) it is known that cooled electrons have steeper distribution functions than uncooled ones, i.e. cooled electrons have an energy dependance that is proportional to $\gamma^{-p-1}$, while uncooled ones have a $\gamma^{-p}$ dependance, i.e. they still retain the spectrum at injection.

High energy electrons can also lose energy by inverse Compton scattering on ambient photons. These photons can illuminate the source of electrons externally or they can be the synchrotron photons mentioned earlier. In this latter case the process is called synchro-self compton. The inclusion of the inverse Compton process has two important results: (a) It will produce a high energy spectral component in the photon spectrum and; (b) depending on the respective magnetic and photon field energy densities, it can alter the electron distribution function, thus affecting directly the shape of the radiated photon spectrum.

\subsection{Comparisons to blazar models}

The radiation coming from the GRB shock, as was described above, has the same underlying physical principles as the socalled "one-zone" SSC AGN leptonic models set forward to explain the MW spectrum of blazar emission (Inoue \& Takahara 1996; Mastichiadis \& Kirk 1997). These models, based on earlier ideas set by Maraschi et al. (1992) and Bloom \& Marscher (1996), address essentially the same problem of electron injection, cooling and photon radiation. However they do not take a "ready" electron distribution but, rather, they obtain it from the solution of a kinetic equation which contains injection, radiative losses, physical escape from the source and possibly reinjection of particles as secondaries from photon-photon absorption. The electron equation is coupled to an equation for photons that has the usual synchrotron and inverse Compton emissivities written in a way as to match the radiative electron losses. It can also have extra terms such as synchrotron self-absorption, photon-photon pair production, etc.

This kinetic equation approach has the advantage that it is self-consistent, i.e. the power lost by the electrons is radiated by 
the photons. Moreover the photon energy density can be calculated at each instant and this feeds back though the SSC losses on the electron equation, thus this approach can treat the SSC intrinsic non-linearity.

Therefore it would have been instructive for us to construct a similar approach to model the radiation of GRB afterglows. However, in GRBs, despite the physical analogies to AGNs, the situation has some obvious differences which come mainly from the hydrodynamics of the GRB outflows, as was outlined in Sect. 2.1:

1. AGN modeling usually involves stationary states. This means that the the radius of the source $R_{\mathrm{s}}$, the Lorentz factor $\Gamma$ and the magnetic field strength $B$ are all considered constant. This holds even when short flares are modeled in a time-dependent way (Mastichiadis \& Kirk 1997; Krawczynski et al. 2002; Katarzyński et al. 2005); as we mentioned earlier, in GRBs all of the above are functions of the distance $r$ from the origin of the explosion;

2. in GRBs, once the profile of the external density is set, then the injection of electrons has at least an upper limit as $\epsilon_{\mathrm{e}}$ cannot exceed unity. There is no such constraint for AGNs as the injected power is essentially a free parameter.

These differences imply that the numerical codes developed for blazars cannot be used as they are for the GRB afterglows, but they have to be modified to take the above into account. We will present such a code in Sect. 4. However we first show some analytical solutions that we have derived for the coupled hydrodynamic-radiation problem.

\section{Analytical solutions}

In this section we will first present the kinetic equation for the electron distribution function, then we will show the solutions for different magnetic field configurations assuming that synchrotron losses dominate and we will end by using these solutions to derive the slope of the synchrotron and SSC lightcurves that corresponds to each B-field configuration considered.

\subsection{Kinetic equation of electrons}

The equation that governs the electron distribution is:

$$
\frac{\partial \tilde{N}(\gamma, t)}{\partial t}+\frac{\partial}{\partial \gamma}(\tilde{b}(\gamma, t) \tilde{N}(\gamma, t))=\tilde{Q}(\gamma, t),
$$

where $\tilde{N}(\gamma, t)$ is the number of electrons having Lorenz factors between $\gamma, \gamma+\mathrm{d} \gamma$ at a time $t$ as measured in the comoving frame. The second term of the left hand side takes into account energy losses of the relativistic electrons due to synchrotron emission and inverse compton scattering in general. The term on the right hand side of the equation describes electron injection. Equation (10) can be also expressed in terms of the distance $r$ measured in the framework of the explosion, instead of $t$, through $\mathrm{d} t \approx \frac{\mathrm{d} r}{c \Gamma(r)}$ :

$\frac{\partial N(\gamma, r)}{\partial r}+\frac{1}{c \Gamma} \frac{\partial}{\partial \gamma}(b(\gamma, r) N(\gamma, r))=\frac{\tilde{Q}}{c \Gamma} \equiv Q(\gamma, r)$

where $N(\gamma, r)$ is now the number of electrons having a Lorenz factor between $\gamma, \gamma+\mathrm{d} \gamma$ at a radius $r$ as measured in the frame of the explosion. In order to obtain some analytical results of the above equation we consider only synchrotron losses. We assume that a fraction $\epsilon_{\mathrm{e}}$ of the accreted kinetic energy is injected into non thermal electrons. The injection of the relativistic electrons can be modeled as a power-law with arbitrary choices of minimum and maximum Lorentz factors in energy:

$Q(\gamma, r)=q_{0}(r) \gamma^{-p} \Theta\left(\gamma-\gamma_{\min }\right) \Theta\left(\gamma_{\max }-\gamma\right) \Theta\left(r-r_{0}\right)$.

At each radius the normalization of the electron distribution is given by the prescribed fraction of the power available as bulk kinetic energy. Equation (8) leads to

$q_{0}(r)=k_{\mathrm{e}} \frac{\left(\Gamma^{2}-\Gamma\right) r^{2}}{c \Gamma}$.

The constant $k_{\mathrm{e}}$ which appears in Eq. (13) is given by:

$k_{\mathrm{e}}= \begin{cases}\epsilon_{\mathrm{e}} \frac{4 \pi \rho_{0} c^{3}}{m_{\mathrm{e}} c^{2}\left(\frac{\gamma_{\min }^{-p+2}-\gamma_{\max }^{-p+2}}{p-2}-\frac{\gamma_{\min }^{-p+1}-\gamma_{\max }^{-p+1}}{p-1}\right)} & \text { if } p \neq 2 \\ \epsilon_{\mathrm{e}} \frac{4 \pi \rho_{0} c^{3}}{m_{\mathrm{e}} c^{2}\left(\ln \left(\frac{\gamma_{\max }}{\gamma_{\min }}\right)+\left(\frac{1}{\gamma_{\max }}-\frac{1}{\gamma_{\min }}\right)\right)} & \text { if } p=2 .\end{cases}$

\subsubsection{Magnetic field of the form $B(r)=B_{0} r^{-3 / 2}$}

As was briefly discussed in Sect. 2, this type of B field is the one customarily adopted for GRB afterglows. Equation (9) implies that a fraction $\epsilon_{\mathrm{B}}$ of the accumulated mass on the RBW goes to amplify the B-field. As $\Gamma \propto r^{-3 / 2}$ in the decelerating phase (cf. Eq. (4)) the above prescription for the B-field is derived at once. Thus Eq. (9) can be further written

$B=B_{0} r^{-3 / 2}$

with

$B_{0}=\left(32 \pi n m_{\mathrm{p}} \epsilon_{\mathrm{B}} c^{2}\right)^{1 / 2} \tilde{\Gamma}$

where the constant $\tilde{\Gamma}$ is defined in Eq. (5). Then the term of synchrotron losses becomes

$b(\gamma, r)=-\alpha_{0} \frac{\gamma^{2}}{r^{3}}$

where $\alpha_{0}=\frac{\sigma_{T} B_{0}^{2}}{6 \pi c m_{\mathrm{e}}}$. The solution of Eq. (11) has a simple expression in the case of $p=2$

$N(\gamma, r)=\frac{2 k_{\mathrm{e}} \tilde{\Gamma}}{3 c \gamma^{2}} K(\gamma, r)$

where

$K(\gamma, r)= \begin{cases}r^{3 / 2}-r_{0}^{3 / 2} & \text { if } \gamma \leq \gamma_{\mathrm{b}} \\ r^{3 / 2}-\left(\frac{1}{\sqrt{r}}+\frac{c \tilde{\Gamma}}{2 \alpha_{0}}\left(\frac{1}{\gamma}-\frac{1}{\gamma_{\max }}\right)\right)^{-3} & \text { if } \gamma>\gamma_{\mathrm{b}}\end{cases}$

and

$\gamma_{\mathrm{b}}=\frac{1}{\frac{1}{\gamma_{\max }}+\frac{2 \alpha_{0}}{c \tilde{\Gamma}}\left(\frac{1}{\sqrt{r_{0}}}-\frac{1}{\sqrt{r}}\right)}$ 


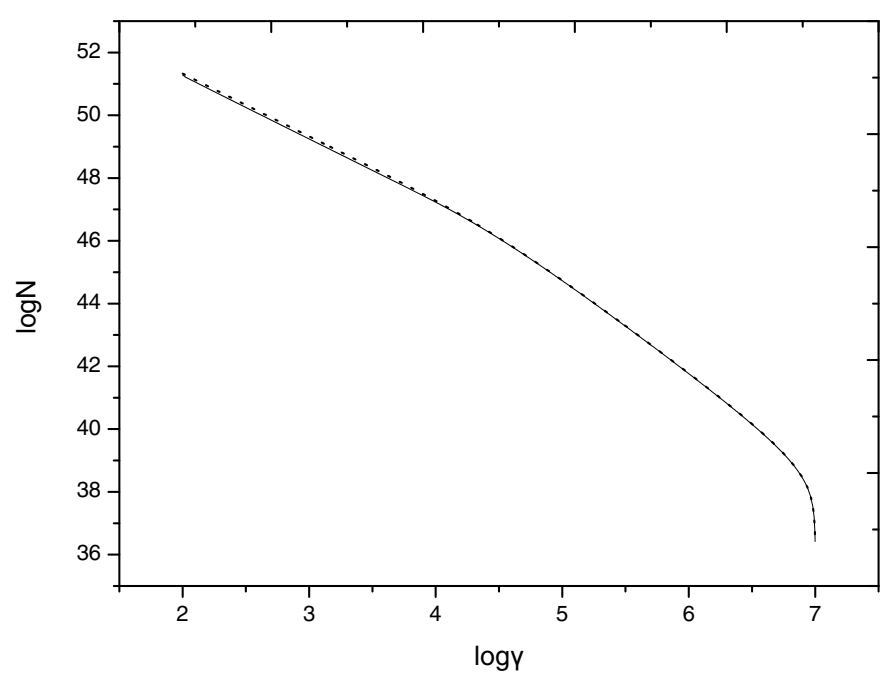

Fig. 1. Comparison between analytical (solid line) and numerical (dotted line) solution in the case of $p=2$ at radius $r=1.5 \times 10^{17} \mathrm{~cm}$ or at observer time $2.7 \times 10^{2} \mathrm{~s}$. There is practically no difference between the two solutions. The parameters used for this plot are: $\epsilon_{\mathrm{e}}=0.1, \epsilon_{\mathrm{B}}=$ $0.001, n=1 \mathrm{~cm}^{-3}, \Gamma_{0}=400, E_{0}=10^{53} \mathrm{erg}, \gamma_{\min }=10^{2}, \gamma_{\max }=10^{7}$

We point out that the second branch of the above solution does not necessarily describe a cooled electron distribution. Only if the relation

$$
\frac{c \tilde{\Gamma} \sqrt{r}}{2 \alpha_{0} \gamma} \ll 1
$$

holds, then the electron distribution can be considered cooled, i.e. $N \propto \gamma^{-3}$. A more physical approach is to consider an electron with Lorentz factor $\gamma_{\mathrm{c}}$, which cools on a timescale equal to the dynamical $t_{\text {dyn }} \approx \frac{2}{5} \frac{r}{c \Gamma}$ (Panaitescu \& Kumar 2000). This is given by:

$\gamma_{\mathrm{c}} \approx \frac{6 \pi m_{\mathrm{e}} c}{\sigma_{\mathrm{T}} B^{2} t_{\mathrm{dyn}}}$.

Then, electrons with Lorentz factors greater than $\gamma_{c}$ cool sufficiently. Condition (19), which arose mathematically, is equivalent to $\gamma \gg \gamma_{c}$. This condition and relation (19) differ only by a factor of 5. From the above discussion it is clear that the Lorentz factor $\gamma_{\mathrm{b}}$ must not be confused with $\gamma_{\mathrm{c}}$, that determines at which Lorentz factor the cooling of the distribution becomes dominant. An example is shown in Fig. 1, where the change of the slope in the power law begins at $\gamma \approx 5 \times 10^{4}$. For the parameters used, $\gamma_{\mathrm{c}}=5.2 \times 10^{4}$ and $\gamma_{\mathrm{b}}=2.8 \times 10^{2}$. In the case where $p \neq 2$ the integral $I_{\mathrm{p}}$ can only be estimated in two asymptotic regimes. The complete calculations and approximations can be found in the Appendix. Here we present the expressions for the electron distribution in these regimes.

In the uncooled regime the relation $\gamma \ll \gamma_{\mathrm{c}}$ holds and the solution takes the form:

$N_{\text {uncooled }} \approx \frac{2 k_{\mathrm{e}} \tilde{\Gamma}}{3 c} r^{3 / 2} \gamma^{-p}$

As we mentioned above, in the cooled regime the condition $\gamma \gg$ $\gamma_{\mathrm{c}}$ holds. Then

$N_{\text {cooled }} \approx \frac{k_{\mathrm{e}} \tilde{\Gamma}^{2}}{\alpha_{0}(p-1)} r^{2} \gamma^{-p-1}$.

\subsubsection{Magnetic field of the form $B(r)=B_{0} \frac{r_{0}}{r}$}

This type of magnetic field might also be related to GRB outflows (see e.g. Vlahakis \& Königl 2003). Here we present the analytical solutions of Eq. (11) for this type of magnetic field, while the complete calculations can be found in the Appendix. Again, if $p=2$ the solution has the simple form

$N(\gamma, r)=\frac{2 k_{\mathrm{e}} \tilde{\Gamma}}{3 c \gamma^{2}} K(\gamma, r)$

where

$K(\gamma, r)= \begin{cases}r^{3 / 2}-r_{0}^{3 / 2} & \text { if } \gamma \leq \gamma_{\mathrm{b}} \\ r^{3 / 2}-\left(\sqrt{r}-\frac{c \tilde{\Gamma}}{2 \alpha_{0}}\left(\frac{1}{\gamma}-\frac{1}{\gamma_{\max }}\right)\right)^{3} & \text { if } \gamma>\gamma_{\mathrm{b}}\end{cases}$

and

$\gamma_{\mathrm{b}}=\frac{1}{\frac{1}{\gamma_{\max }}+\frac{2 \alpha_{0}}{c \tilde{\Gamma}}\left(\sqrt{r}-\sqrt{r_{0}}\right)}$

The constant $\alpha_{0}$ is given by

$\alpha_{0}=\frac{\sigma_{T} B_{0}^{2} r_{0}^{2}}{6 \pi c m_{\mathrm{e}}}$

If $p>2$ then the solution is found in two regimes, as in (3.1.1).

The uncooled part of the electron distribution is given by:

$N_{\text {uncooled }} \approx \frac{2 k_{\mathrm{e}} \tilde{\Gamma}}{3 c} r^{3 / 2} \gamma^{-p}$

which is exactly the same as the one calculated for the magnetic field $B \propto r^{-3 / 2}$. In this regime the cooling timescale of electrons is much greater than the dynamical timescale. Thus, this part of the electron distribution will not be affected by a different type of magnetic field which is the cause of electron cooling.

The situation is different for the part of the distribution where cooling is dominant:

$N_{\text {cooled }} \approx \frac{k_{\mathrm{e}} \tilde{\Gamma}^{2}}{\alpha_{0}(p-1)} r \gamma^{-p-1}$.

\subsubsection{Constant magnetic field $B_{0}$}

In order to check our analytical results we have also solved Eq. (11) for the case of a constant magnetic field. This calculation has already been done by Dermer \& Chiang (1998) and thus we can compare our results with theirs. The outline of the comparison can be found in the Appendix (A.3). In the uncooled regime the solution is given by:

$N_{\text {uncooled }} \approx \frac{2 k_{\mathrm{e}} \tilde{\Gamma}}{3 c} r^{3 / 2} \gamma^{-p}$.

The above expression for the uncooled part of the electron distribution is again the same as for the other types of magnetic field presented in the previous sections. In the cooled regime

$N_{\text {cooled }} \approx \frac{k_{\mathrm{e}} \tilde{\Gamma}^{2}}{\alpha_{0}(p-1)} r^{-1} \gamma^{-p-1}$.

In this case the cooled part of the distribution reduces as the radius of the blast wave increases. This behavior of the cooled part of the distribution differs from the one presented in the previous sections where the total number of electrons within the shell increased with increasing radius. 
Table 1. Negative of slopes of time profiles.

\begin{tabular}{llll}
\hline \hline & $B=$ Const. & $B \propto r^{-1}$ & $B \propto r^{-3 / 2}$ \\
\hline$\alpha_{\mathrm{s}}^{\text {uncooled }}$ & $\frac{3 p-3}{16}$ & $\frac{5 p-1}{16}$ & $\frac{3 p}{8}$ \\
$\alpha_{\mathrm{s}}^{\text {cooled }}$ & $\frac{3 p+10}{16}$ & $\frac{5 p+4}{16}$ & $\frac{6 p+1}{16}$ \\
$\alpha_{\mathrm{ssc}}^{\text {uncooled }}$ & $\frac{3 p-1}{16}$ & $\frac{5 p+1}{16}$ & $\frac{3 p+1}{8}$ \\
$\alpha_{\mathrm{ssc}}^{\text {cooled }}$ & $\frac{3 p+22}{16}$ & $\frac{5 p+8}{16}$ & $\frac{6 p+1}{16}$ \\
\hline
\end{tabular}

\subsection{Analytic flux time profiles}

The kinetic equation of the electron distribution is being solved in the comoving frame, as shown in Sect. (3.1). Synchrotron and SSC spectra are also first calculated in the comoving frame and then transformed into the observer frame. For this we use a relation which connects time in the observer frame and radius $r$ which appears in all our analytical solutions. Thus,

$t_{\mathrm{obs}} \approx \int_{r_{0}}^{r} \frac{\mathrm{d} r}{2 c \Gamma^{2}}$

where $r$ is the radius of the blast wave measured in the comoving frame. If the distance of the source from the observer is $D$ then the respective synchrotron and SSC fluxes at the observer frame are given by:

$F_{\mathrm{s}}\left(v_{\mathrm{obs}}, t_{\mathrm{obs}}\right)=\frac{J_{\mathrm{s}}\left(v_{\mathrm{obs}}, t_{\mathrm{obs}}\right) \Gamma}{4 \pi D^{2}}$

$F_{\mathrm{ssc}}\left(v_{\mathrm{obs}}, t_{\mathrm{obs}}\right)=\frac{J_{\mathrm{ssc}}\left(v_{\mathrm{obs}}, t_{\mathrm{obs}}\right) \Gamma}{4 \pi D^{2}}$

where $J_{\mathrm{s}}, J_{\mathrm{ssc}}$ are the synchrotron and SSC power per unit frequency emitted in the comoving frame. It is interesting to examine the dependence of the observed fluxes on time, in cases where the electron kinetic equation can be analytically solved. Thus, we consider the cases that we have treated in (3.1). Since we are interested only in the time dependency, in what follows we can work using proportionalities. form

Equations (31) and (32) can be reduced to the simplified

$F_{\mathrm{S}} \propto C_{\mathrm{e}} \Gamma^{1+\alpha} B^{\frac{p+1}{2}}$

$F_{\mathrm{ssc}} \propto \frac{C_{\mathrm{e}}{ }^{2}}{r^{2}} \Gamma^{1+\alpha} B^{\frac{p+1}{2}}$

where $C_{\mathrm{e}}=C_{\mathrm{e}}(r)$ is the normalization factor of the electron distribution. After the deceleration radius (Eq. (6)) it is straightforward to show that $t_{\mathrm{obs}} \propto r^{4}$. Thus the synchrotron and SSC fluxes can be expressed as power laws of the observed time with exponents $\alpha_{\mathrm{s}}, \alpha_{\mathrm{ssc}}$ respectively. The exponents are found in the asymptotic regimes of uncooled and cooled electron distributions and are presented in Table 1 . The slopes of the synchrotron flux time profiles in the non-radiative regime (see Table 1) coincide with the respective ones presented by Dermer \& Chiang (1998). Table 1 shows that SSC flux time profiles are steeper than the respective synchrotron profiles both in the uncooled and cooled regime, for all the magnetic field configurations discussed in Sect. 3.1.

\section{Numerical approach}

\subsection{The code}

The equation solved in the previous section forms the basis of the electron kinetic equation; we proceed now to augment this with more processes and to solve it numerically. Since SSC losses depend on the synchrotron photon energy density, we have to write an accompanying equation for photons which is coupled to the electron equation. Similar types of equations have been solved for the blazar (Mastichiadis \& Kirk 1997) and prompt/early afterglow GRB cases (Mastichiadis \& Kazanas 2009) - note that in this latter case a third equation for protons was added.

Assuming, as before, that the electrons are a function of distance from the center of the explosion and energy, their equation reads

$\frac{\partial n_{\mathrm{e}}}{\partial r}+\mathcal{L}_{\mathrm{e}}^{\mathrm{syn}}+\mathcal{L}_{\mathrm{e}}^{\mathrm{ics}}+\mathcal{L}_{\mathrm{e}}^{\mathrm{ad}}=Q_{\mathrm{e}}^{\mathrm{inj}}+Q_{\mathrm{e}}^{\gamma \gamma}$

while the corresponding photon equation is

$\frac{\partial n_{\gamma}}{\partial r}+\frac{c n_{\gamma}}{t_{\mathrm{cr}}}+\mathcal{L}_{\gamma}^{\gamma \gamma}+\mathcal{L}_{\gamma}^{\mathrm{ssa}}=Q_{\gamma}^{\mathrm{syn}}+Q_{\gamma}^{\mathrm{ics}}$.

The operators $\mathcal{L}$ denote losses and escape from the system while $Q$ denote injection and source terms. The unknown functions $n_{\mathrm{e}}$ are $n_{\gamma}$ are the differential number densities of electrons and photons respectively and the physical processes which are included in the kinetic equations are: (1) electron synchrotron radiation and synchrotron self absorption (denoted by the superscripts "syn" and "ssa" respectively); (2) inverse Compton scattering ("ics"); (3) photon-photon pair production (" $\gamma \gamma$ ") and (4) adiabatic losses ("adi").

The numerical code keeps the same philosophy, as far as the physical processes are concerned, as the one described in Mastichiadis \& Kirk (1995) (hereafter MK95). However, since various modifications have been introduced, we summarize briefly the expressions used:

(i) Synchrotron radiation: (a) The electron loss term $\mathcal{L}_{\mathrm{e}}^{\text {syn }}$ is given by expression (34) of MK95. (b) The photon emissivity term $Q_{\gamma}^{\text {syn }}$ is given using the full high energy emissivity term (see, e.g. Blumenthal \& Gould 1970) instead of the delta-function approximation used in MK95;

(ii) Synchrotron-self absorption: (a) The photon absorption term $\mathcal{L}_{\gamma}^{\text {ssa }}$ is used as in MK95 (Eq. (39)). (b) There is no matching term for electron heating due to this process. However, as synchrotron self absorption is expected to be minimal for the parameters which are of interest here, the error introduced by this omission is expected to be negligible;

(iii) Inverse compton scattering: (a) The electron loss term $\mathcal{L}_{\mathrm{e}}^{\text {ics }}$ is given by solving Eq. (5.7) of Blumenthal \& Gould (1970). (b) The photon emissivity term $Q_{\gamma}^{\text {ics }}$ uses relation (2.48) of the same paper;

(iv) Photon-photon pair production: (a) The electron injection term $Q_{\mathrm{e}}^{\gamma \gamma}$ is given by expression (57) of MK95. (b) The photon absorption term $\mathcal{L}_{\gamma}^{\gamma \gamma}$ is given by expression (54) of MK95;

(v) Electron injection: the quantity $Q_{\mathrm{e}}^{\mathrm{inj}}$ is the electron injection rate which can take any functional form of distance $r$ and energy $\gamma$. Following the usually assumed case, we take it to be of a power-law form as given by Eq. (12). The power-law 


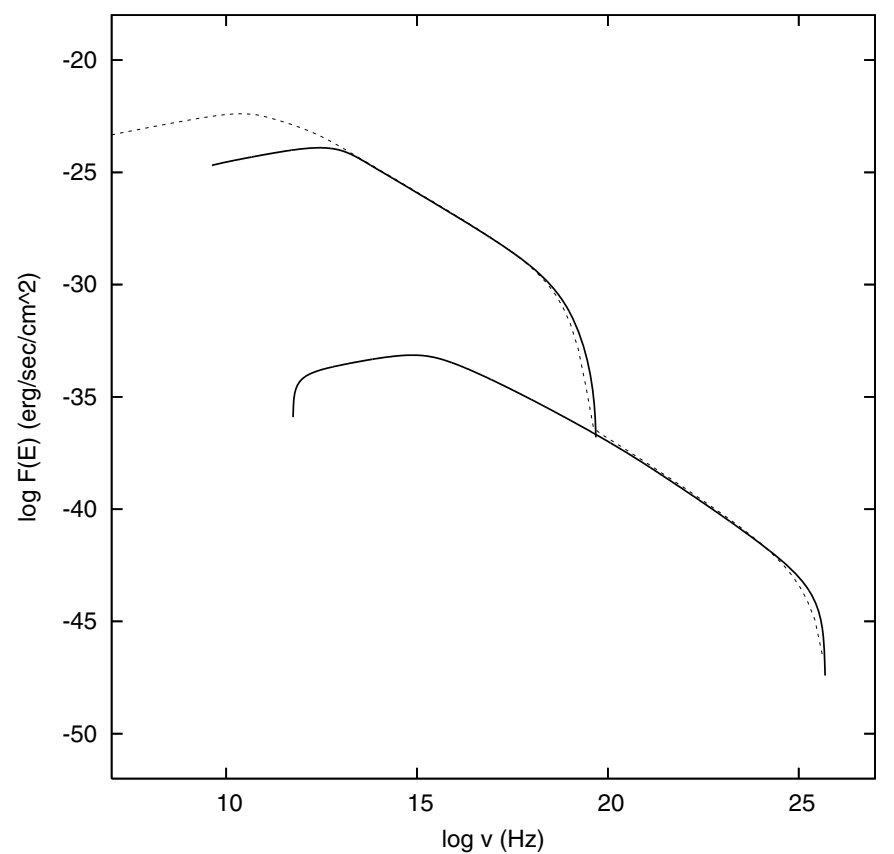

Fig. 2. Synchrotron and SSC spectra for a case with $\Gamma_{0}=100, E_{0}=$ $10^{53} \mathrm{erg}, R_{0}=10^{14} \mathrm{~cm}, n=1 \mathrm{~cm}^{-3}, p=2, \gamma_{\min }=10, \gamma_{\max }=10^{4}$ and $B(r)=10^{6}\left(\frac{R_{0}}{r}\right)$ Gauss. The spectra are calculated at $r=2 \times 10^{17} \mathrm{~cm}$. The source was assumed at a distance of $D=3 \mathrm{Gpc}$. Analytical results are depicted with the full line, while the numerical ones with the dotted one. The difference in the low part of the spectrum arises from the fact that in the analytical results we did not solve for the electron distribution below $\gamma_{\min }$. In the numerical case we did not impose such restriction. For comparison we ignored SSC losses in the numerical code.

index, the normalization and the upper and lower energy cutoffs can be treated as free parameters;

(vi) Photon escape: this is characterized by the light crossing time of the source $t_{\mathrm{cr}}=R_{\mathrm{s}} / c$ in the comoving frame. This is not constant but changes according to the relation $R_{\mathrm{s}}=r / \Gamma$.

There is still one more free parameter to be addressed in specifying the code quantities and this is the behavior of the magnetic field with radius. While it is trivial to adopt any r-dependence we will use the standard case $B \propto r^{-3 / 2}$ except in one of the tests that follow.

\subsection{Tests}

There are various tests that we have performed to test the validity of the code. As the various rates of the radiative processes have essentially the same form as the ones used in the past to model AGN MW emission (Mastichiadis \& Kirk 1997; Konopelko et al. 2003), they have been checked many times against the results of e.g. Coppi (1992); Katarzyński et al. (2005) and others.

The new aspect introduced in the code is its dynamical behavior and in order to test this we have checked the code extensively against the analytical results (spectral shape and lightcurve slopes) derived in the previous section. As an example we show in Fig. 2 such a comparison of the multiwavelength spectra derived with the analytical method of the previous section with the results of the numerical code.

The other major test was to compare our results with results already published in the literature, such as the ones given in

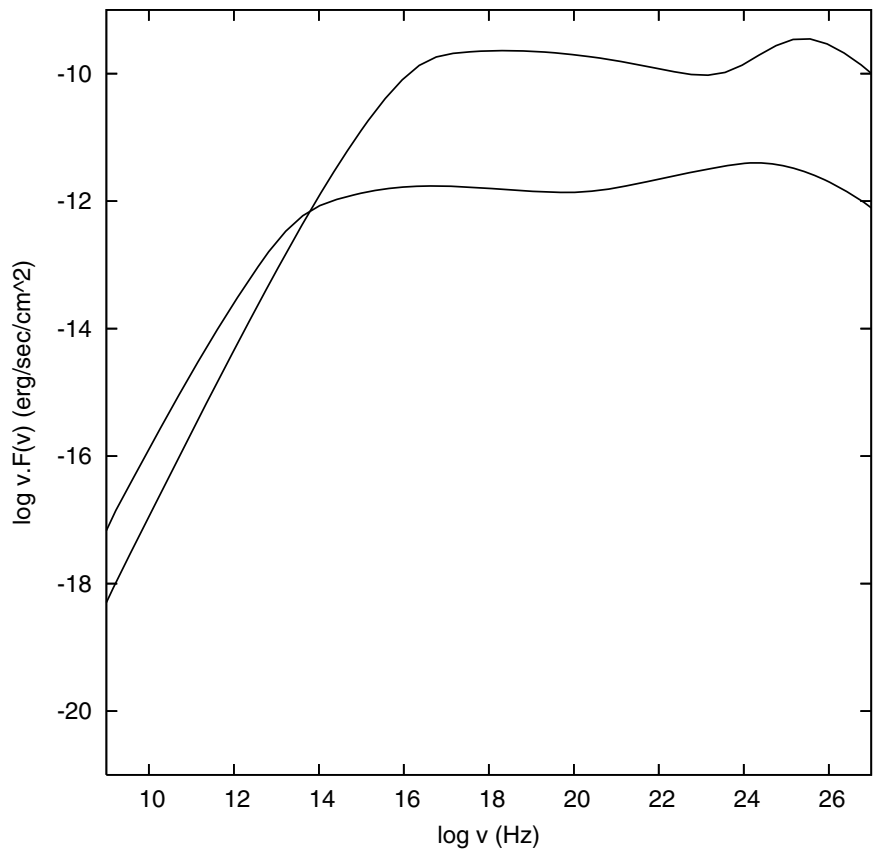

Fig. 3. Multiwavelength spectra with parameters similar to the ones stated in Fig. 4b of Fan et al. (2008). These should be compared with the two top curves of the aforementioned figure.

(Fan et al. 2008). Figure 3 reproduces two curves of Fig. $4 \mathrm{~b}$ of the aforementioned paper with very good agreement.

\section{Results}

In what follows we apply the numerical code to examine the dynamical evolution of the MW GRB afterglow spectra. For this we use the standard afterglow adopted parameters, i.e. the magnetic field is given by rel. (9) with $\epsilon_{\mathrm{B}}$ a free parameter, while the injected electron luminosity is controlled by $\epsilon_{\mathrm{e}}$ which is also treated as a free parameter. Furthermore we adopt a value for $\gamma_{\min }$ that is not constant but is of the form (Sari et al. 1998)

$\gamma_{\min }=\frac{p-2}{p-1} \epsilon_{\mathrm{e}} \frac{m_{p}}{m_{\mathrm{e}}} \Gamma$

Our aim is to see whether (i) SSC losses can modify the electron distribution function, and therefore, the photon spectrum and; (ii) photon-photon absorption, a process that has been neglected thus far, can be of some importance, not only taken as a $\gamma$-ray absorption mechanism but also as an electron (and positron) reinjection one.

As a first case we show an example for typical values assumed usually for GRB afterglows. Figure 4 shows the photon spectrum obtained at radius $R=3.4 \times 10^{17} \mathrm{~cm}$ for $\Gamma_{0}=400$, $n=1 \mathrm{~cm}^{-3}, \epsilon_{\mathrm{e}}=.1, \epsilon_{\mathrm{B}}=.001$. The electrons were assumed to have a power-law distribution with slope $p=2.3$ while their maximum cutoff was taken to be constant and equal to $\gamma_{\max }=4 \times 10^{7}$. Here and in the next Figures the GRB was set at $z=1$. Note also that we assume that the evolution of the Lorentz factor $\Gamma$ follows the adiabatic prescription implied by Eq. (2) and that we have not taken into account any attenuation for TeV $\gamma$-rays due to absorption on the IR background. The full line curve depicts the photon spectrum when all processes are included, the dashed line one when photon-photon absorption is left out and the dotted line one when inverse Compton 


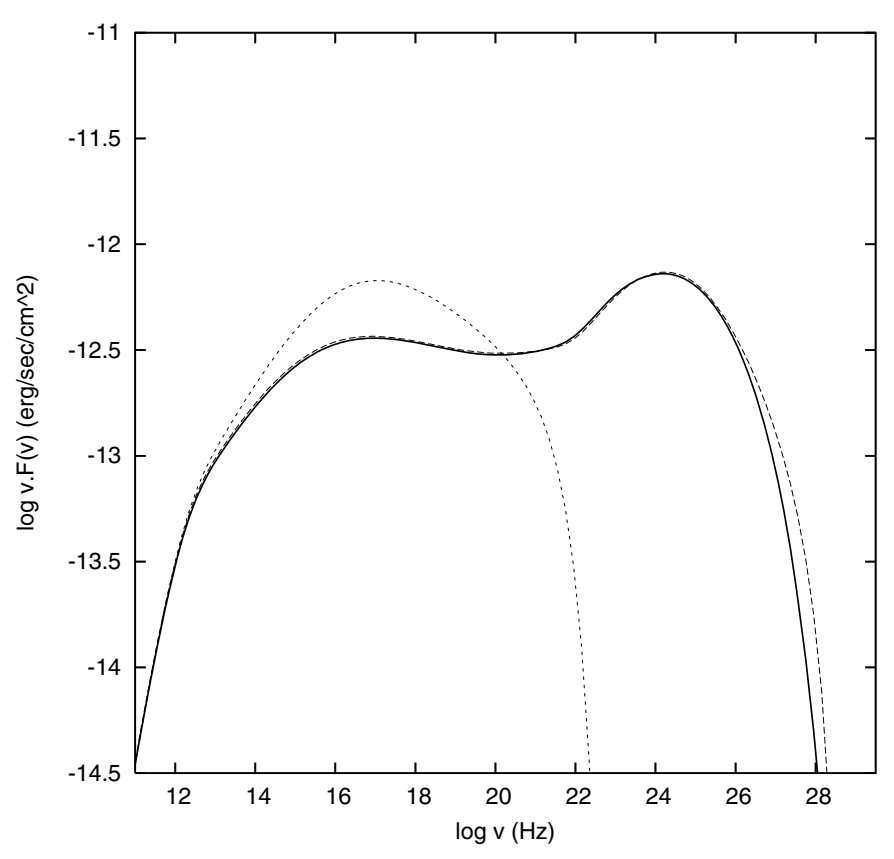

Fig. 4. Photon spectrum obtained at radius $R=3.4 \times 10^{17} \mathrm{~cm}$ or equivalently at observer time $t_{\mathrm{obs}}=7 \times 10^{3} \mathrm{~s}$ for $\Gamma_{0}=400, E_{0}=10^{53} \mathrm{erg}$, $n=1 \mathrm{~cm}^{-3}, \epsilon_{\mathrm{e}}=.1, \epsilon_{\mathrm{B}}=.001$. The electrons were assumed to have a power-law distribution with slope $p=2.3$ while their maximum cutoff was $\gamma_{\max }=4 \times 10^{7} \cdot \gamma_{\min }$ was given by expression (37). The full line corresponds to the spectrum when all processes are included, the short-dashed one when $\gamma \gamma$ is omitted and the dotted line one when only synchrotron is included.

scattering is also omitted both as emission in the photon equation and as a loss mechanism in the electron equation, i.e. this case can be considered as pure synchrotron. Although this latter case is clearly an oversimplification, we have included it for comparison. One can see that $\gamma \gamma$ absorption influences only the highest part of the spectrum by making it steeper. Pair reinjection does not significantly alter the lower spectral parts because only a very small fraction of the energy has been absorbed and is thus available for redistribution. On the other hand, SSC losses have an impact on the spectrum. This effect can be seen better in Fig. 5 which shows the electron distribution function with (full lines) and without (dashed lines) SSC losses at the aforementioned radius. As in standard theory, the braking energy $\gamma_{\mathrm{c}}$ divides the cooled $\left(\gamma>\gamma_{\mathrm{c}}\right)$ from the uncooled $\left(\gamma<\gamma_{\mathrm{c}}\right)$ part of the electrons. A first comment one could make is that the synchrotron break does not appear as a sharp turnover but as a gradual one which affects the power law index of electrons by at least one order of magnitude around $\gamma_{\mathrm{c}}$. A second comment is that inclusion of SSC losses changes the "pure" synchrotron picture. The electron distribution function becomes flatter and this is a result of the SSC losses. The specific shape can be explained because the SSC losses at each electron energy consist of both losses in the Thomson and the Klein Nishina regime. As the electron energy increases, the fraction of Klein Nishina to Thomson losses also increases, with the result that the total SSC losses are reduced.

\subsection{Dynamical evolution}

Figure 6 shows snapshots of photon spectra obtained for the same parameters as above at three different radii: $R=R_{\mathrm{dec}}$, $R=3.2 R_{\mathrm{dec}}$ and $R=10 R_{\mathrm{dec}}$. As before, full line curves depict the spectrum when all processes are included, dashed line ones

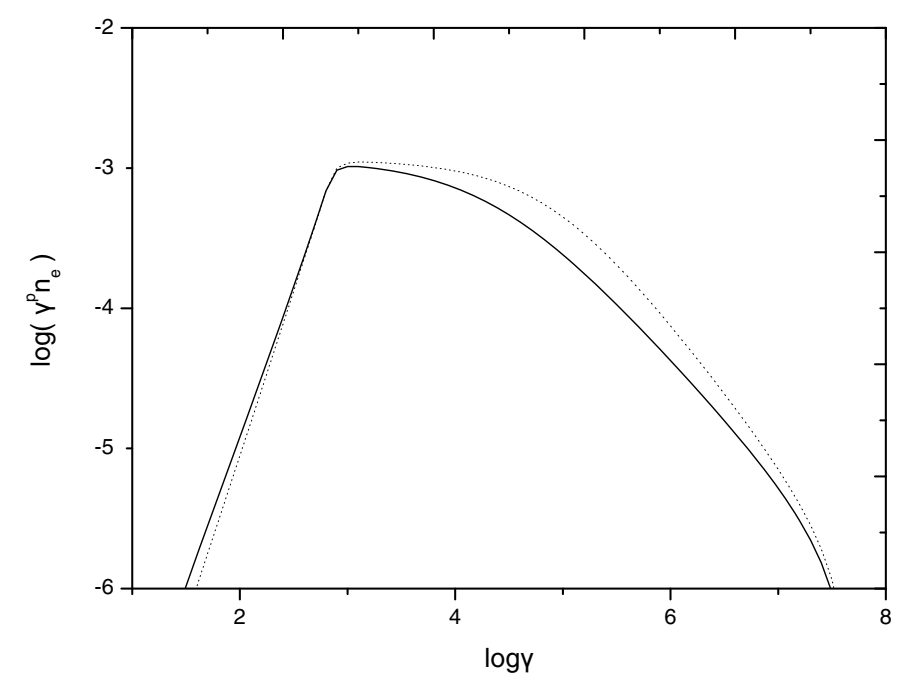

Fig. 5. Electron distribution function for $p=2.3$, normalized by the factor $\sigma_{\mathrm{T}} m_{\mathrm{e}} c^{2} R_{\mathrm{S}}$ and multiplied by $\gamma^{p}$, that corresponds to the photon spectrum of Fig. 4. The full line corresponds to the solution when all processes are included, while the dotted line one when only synchrotron losses are included.

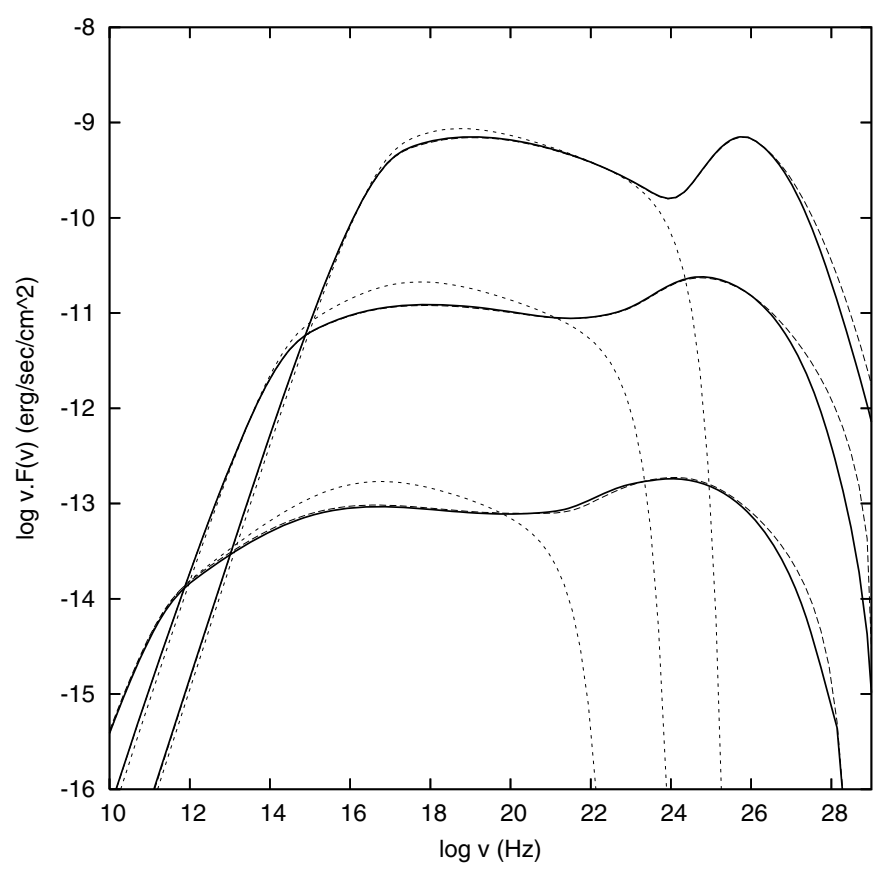

Fig. 6. Multiwavelength GRB afterglow spectra for $\Gamma_{0}=400, E_{0}=$ $10^{53} \mathrm{erg}, n=1 \mathrm{~cm}^{-3}, \epsilon_{\mathrm{e}}=.1, \epsilon_{\mathrm{B}}=.001$, at $R=R_{\mathrm{dec}}, R=3.2 R_{\mathrm{dec}}$ and $R=10 R_{\mathrm{dec}}$ (top to bottom) or at equivalent observer times $t_{\mathrm{obs}}=7.2 \mathrm{~s}$, $2.7 \times 10^{2} \mathrm{~s}, 2.4 \times 10^{4} \mathrm{~s}$ respectively. The electrons were assumed to have a power-law distribution with slope $p=2.3$ and $\gamma_{\max }=4 \times 10^{7}$. Full line curves depict the spectrum when all processes are included, dashed line ones when photon-photon absorption is left out while dotted line ones are pure synchrotron cases.

when photon-photon absorption is left out and dotted line ones when inverse Compton scattering is also omitted. Obviously $\gamma \gamma$ absorption affects only the highest energies and does not play any significant role throughout the evolution.

On the other hand, SSC losses seem to play a role that becomes slightly more important as the radius increases. This can be understood from the fact that as the magnetic field drops, 


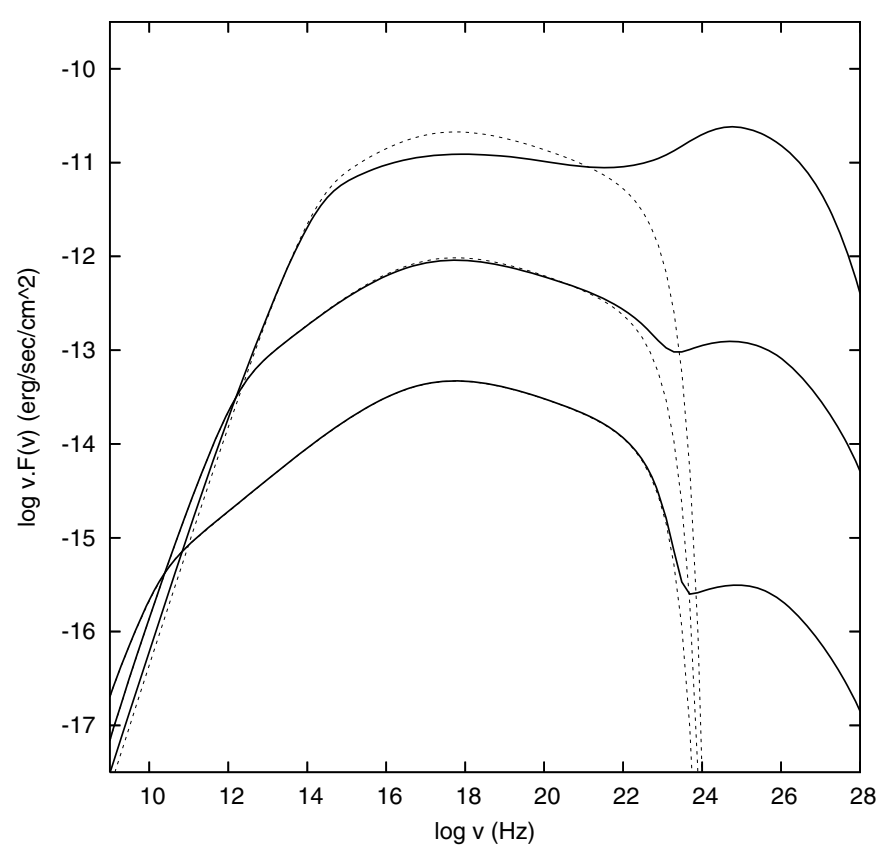

Fig. 7. Multiwavelength GRB afterglow spectra for $\Gamma_{0}=400, E_{0}=$ $10^{53} \mathrm{erg}, n=1 \mathrm{~cm}^{-3}, \epsilon_{\mathrm{B}}=.001$. The electrons were assumed to have a power-law distribution with slope $p=2.3$ and $\gamma_{\max }=4 \times 10^{7}$. All photon spectra are calculated at the radius $R=3.2 R_{\mathrm{dec}}$ or at observer time $t_{\mathrm{obs}}=2.7 \times 10^{2} \mathrm{~s}$ with $\epsilon_{\mathrm{e}}=0.1,0.01$ and 0.001 (top to bottom). Full line curves depict the spectra when all processes are included and dotted line ones depict the corresponding spectra when only synchrotron radiation is taken into account.

two contradicting results occur: one is that synchrotron cooling becomes less efficient (it moves from "fast" to "slow") and the number of available soft photons for upscattering is reduced. However, these same photons become softer with radius (since both $B$ and $\gamma_{\min }$ are reduced outwards) and therefore one expects more collisions in the Thomson regime where electron losses become more efficient. This effect can be seen by comparing the shapes of the MW spectra at the three radii: the shape of the SSC component starts resembling the synchrotron one as the distance increases and the SSC losses are dominated by collisions in the Thomson regime.

\subsection{Role of $\epsilon_{\mathrm{e}}$}

The effect that $\epsilon_{\mathrm{e}}$ has on the spectrum is more straightforward. As $\epsilon_{\mathrm{e}}$ decreases (for fixed $\epsilon_{\mathrm{B}}$ ) the electron spectra are increasingly dominated by synchrotron losses and the effect of SSC losses becomes marginal. This is shown in Fig. 7 which depicts the radiated photon spectra for three values of $\epsilon_{\mathrm{e}}=0.1,0.01$ and 0.001 (top to bottom). Here $\epsilon_{\mathrm{B}}=0.001$, while the rest of the parameters are as in the previous case. In order to avoid confusion we have calculated all spectra at radius $R=3.2 R_{\mathrm{dec}}$. Note that, as $\epsilon_{\mathrm{e}}$ decreases, the SSC component drops as the quadratic of the synchrotron component, a fact that is well known in the SSC AGN models.

\subsection{Role of $\epsilon_{\mathrm{B}}$}

Figure 8 shows the effects that $\epsilon_{\mathrm{B}}$ has on the MW spectra. Here the run has the same parameters as before, however the spectra are calculated at the same radius $R=3.2 R_{\mathrm{dec}}$ with $\epsilon_{\mathrm{B}}=0.1,10^{-3}$ and $10^{-5}$. SSC losses change the spectra only for intermediate

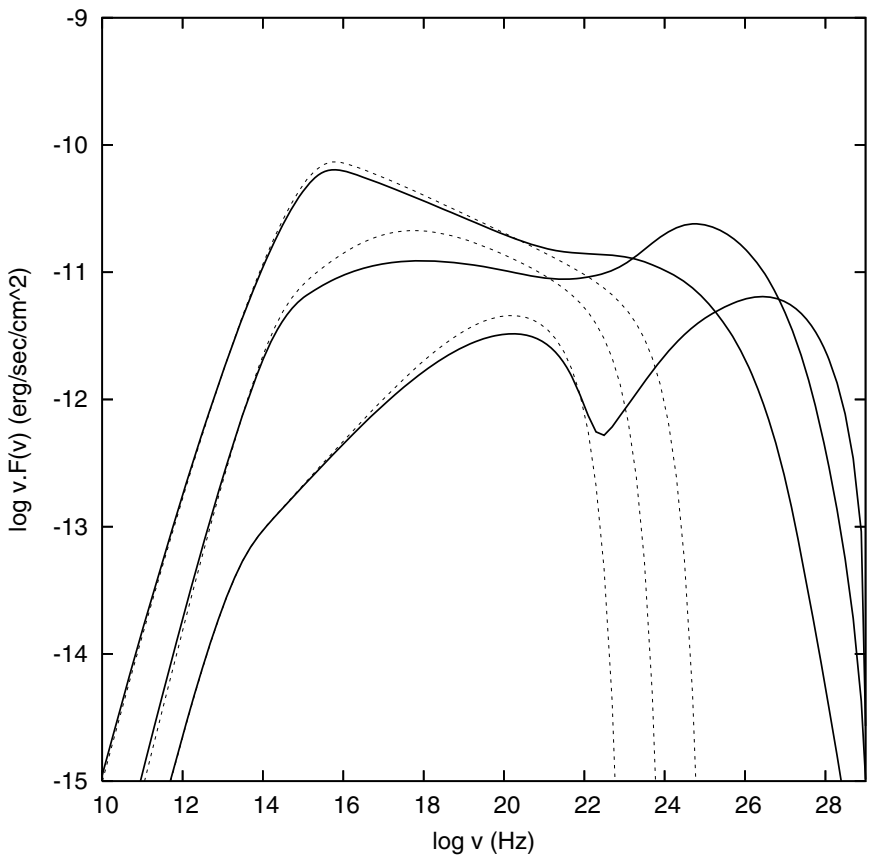

Fig. 8. Multiwavelength GRB afterglow spectra for $\Gamma_{0}=400, E_{0}=$ $10^{53} \mathrm{erg}, n=1 \mathrm{~cm}^{-3}, \epsilon_{\mathrm{e}}=.1$. The electrons were assumed to have a power-law distribution with slope $p=2.3$ and $\gamma_{\max }=4 \times 10^{7}$. All photon spectra are calculated at the radius $R=3.2 R_{\mathrm{dec}}$ or at observer time $t_{\mathrm{obs}}=2.7 \times 10^{2} \mathrm{~s}$ with $\epsilon_{\mathrm{B}}=0.1,10^{-3}$ and $10^{-5}$ (top to bottom). Full line curves depict the spectra when all processes are included and dotted line the corresponding spectra when only synchrotron radiation is taken into account.

values of $\epsilon_{\mathrm{B}}$. High values of $\epsilon_{\mathrm{B}}$ lead to fast cooling and a predominance of synchrotron radiation. On the other hand, low values of $\epsilon_{\mathrm{B}}$ lead to inefficient cooling and only the high synchrotron frequencies are affected by the SSC cooling which occurs in the deep KN regime.

\subsection{Role of external density $n$}

Figure 9 shows the way the MW spectra change in the case when the density is increased to $1000 \mathrm{part} / \mathrm{cm}^{3}$. This figure has to be directly compared to Fig. 4. Inclusion of the SSC losses makes the spectrum depart significantly from the pure synchrotron case. Therefore this is a clear Compton dominated case with the SSC component exceeding the synchrotron one by an order of magnitude. Furthermore $\gamma \gamma$ absorption produces a contribution that affects the entire spectrum as substantial pair injection redistributes the luminosity from the high energy end to the lower.

\section{Summary/discussion}

In the present paper we have applied the "one-zone" SSC model which is customarily applied to the multiwavelength blazar emission of GRB afterglows. For this, we have used a numerical code that treats electron injection and cooling and we have calculated self-consistently the electron distribution and radiated photon spectrum at each radius of the relativistic blast wave. In this sense the present work should be considered as complimentary to Chiang \& Dermer (1999) and Fan et al. (2008).

One difference between the modeling of blazar emission (even in flaring conditions) and GRB afterglows is that in the latter case there is continuous evolution of the bulk relativistic 


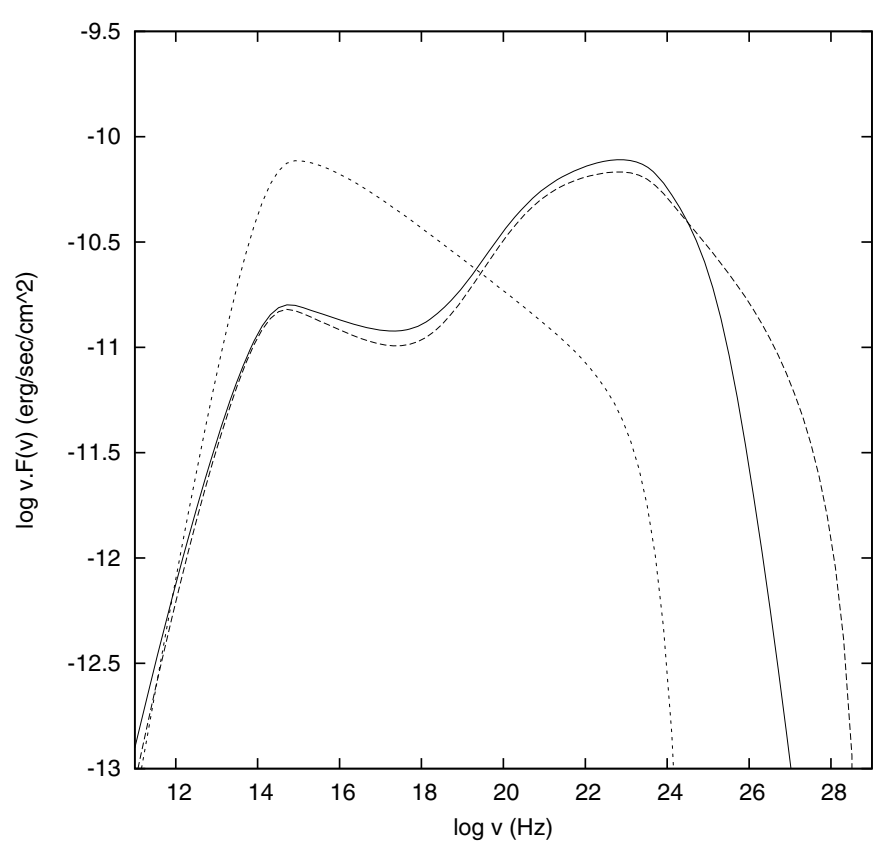

Fig. 9. Multiwavelength GRB afterglow spectra for $\Gamma_{0}=400, E_{0}=$ $10^{53} \mathrm{erg}, n=1000 \mathrm{~cm}^{-3}, \epsilon_{\mathrm{e}}=.1$ and $\epsilon_{\mathrm{B}}=0.001$. The electrons were assumed to have a power-law distribution with slope $p=2.3$ and $\gamma_{\max }=$ $4 \times 10^{7}$. All photon spectra are calculated at the radius $R=2.7 \times 10^{16} \mathrm{~cm}$ or equivalently at observer time $t_{\mathrm{obs}}=2.8 \times 10^{2} \mathrm{~s}$. The full line curve depicts the spectrum when all processes are included, the dashed line one when photon-photon absorption is left out while the dotted line one is a pure synchrotron case. Notice that the inclusion of the SSC losses redistributes the radiated power to high energies while $\gamma \gamma$ has an effect on the entire spectrum through pair injection.

Lorentz factor $\Gamma$, the magnetic field strength $B$ and the radius of the emitting source $R_{\mathrm{s}}=r / \Gamma$. In order to test the numerical code in this new setting, we have solved analytically the kinetic equation of the evolving electrons for the uniform density case under certain simplifying assumptions and compared the resulting electron distribution function and radiated spectra to the ones given from the code for similar parameters. The analytical solutions, details of which can be found in the Appendix, have their own interest despite the introduced simplifications as they can give simple expressions for the dependence of the lightcurve on the spectral index in the case where the losses come solely from the synchrotron mechanism. We find, for example, that the magnetic field prescription plays a significant role in determining the slopes of the flux time profiles (see Table 1). Moreover they correctly treat the synchrotron cooling break which should not be taken as an abrupt change of slope but as a very gradual one.

Restricting ourselves to the uniform density case in the adiabatic approximation, we have also performed numerical runs of the standard GRB afterglow approach as this can be summarized by the introduction of the usual parameters $\epsilon_{\mathrm{B}}$ and $\epsilon_{\mathrm{e}}$. Our aim was two-fold: (i) To see whether GRB afterglows can become Compton dominant, similar to the cases found in modeling AGNs and; (ii) to investigate the effect $\gamma \gamma$ absorption and subsequent pair reinjection might have on the afterglow multiwavelength spectra. Starting from the latter case first we found that, at least for the set of parameters used, $\gamma \gamma$ absorption has an effect, as a photon attenuation mechanism, only at the very high energy part of the spectrum. Therefore it could affect only the potential TeV GRB observations - we point out also that $\mathrm{TeV}$ emission from GRBs at high $\mathrm{z}$ will be subject not only to internal $\gamma \gamma$ attenuation, but also to extra absorption by photons of the IR background, an effect that already has important consequences even for low z TeV blazars (see, e.g. Konopelko et al. 2003) However, as a pair injection mechanism, $\gamma \gamma$ absorption has an effect over the whole spectrum that becomes increasingly important with increasing values of the external density (compare Fig. 4 with Fig. 9).

At the same time, we found that SSC has an impact on the MW spectra as it introduces an extra source of cooling in the electron distribution. As one would expect, it depends also on the parameters $\epsilon_{\mathrm{e}}, \epsilon_{\mathrm{B}}$ and, more critically, on the value of the ambient density $n$. For low values of $\epsilon_{\mathrm{e}}$ it does not practically play any role and the cooling comes solely from synchrotron radiation (see Fig. 7). Thus the GRB afterglow is in the "synchrotron dominated" regime. However, for high values of $\epsilon_{\mathrm{e}}$ it becomes important and this can be seen from the fact that in the $v F_{v}$ spectra the SSC component carries about the same luminosity as the synchrotron component even in the $n=1 \mathrm{~cm}^{-3}$ case. The effect becomes quite severe for higher values of $n$ where the SSC component carries an increasingly great er part of the luminosity (see Fig. 9). These afterglows are clearly Compton dominated.

The fact that we have limited our analysis to the adiabatic case restricts the allowed values of $\epsilon_{\mathrm{e}}$. As a limiting case we have adopted the value $\epsilon_{\mathrm{e}}=0.1$ which introduces a maximum (corresponding to the fast cooling case) error of a few percent in the Lorentz factor $\Gamma$ as this has to be corrected for radiative losses (Chiang \& Dermer 1999). In any case, these corrections are small, they do not affect the spectral shapes and, therefore, they cannot alter the basic results of the present paper.

Concluding we can say that the inclusion of the effects of SSC (not only as an emission, but as a loss process as well) and, to a lesser degree, of $\gamma \gamma$ absorption can have a significant impact on the GRB afterglow multiwavelength spectra. As expected, the overall picture that emanates from these processes is rather complicated. However, as a rule of thumb, we can say that the aforementioned processes become increasingly important for high external densities and high $\epsilon_{\mathrm{e}}$ cases. Their inclusion has some non-trivial consequences on the lightcurves as both the photon spectra slope and the energy break are modified. On the other hand, they can be rather safely neglected for high $\epsilon_{\mathrm{B}}$ and low $\epsilon_{\mathrm{e}}$ or $n$ cases.

Based on the above results, we expect that SSC losses will play an important role in a wind-type density profile, especially at early times when densities are high. This will be the subject of another paper.

Acknowledgements. We would like to thank Prof. T. Piran and Dr. D. Giannios for many interesting discussions and comments on the manuscript. We also thank the referee, Dr. M. Böttcher, for his swift reply and for making points that helped improve the manuscript. This research was funded in part by a Grant from the special Funds for Research (ELKE) of the University of Athens.

\section{Appendix A: Analytical solutions of the electron kinetic equation}

\section{A.1. Magnetic field of the form $B=B_{0} r^{-3 / 2}$}

The solution of Eq. (11) is

$N(\gamma, r)=\int_{-\infty}^{r} \mathrm{~d} \tilde{r} Q(\tilde{\gamma}, \tilde{r}) \frac{\tilde{\gamma}^{2}}{\gamma^{2}}$

or changing the integration variable

$N(\gamma, r)=\frac{1}{\alpha_{0} \gamma^{2}} \int_{\gamma}^{\infty} \mathrm{d} \tilde{\gamma} Q(\tilde{\gamma}, \tilde{r}) c \Gamma(\tilde{r}) \tilde{r}^{3}$. 
The electron distribution is in general given by:

$N(\gamma, r)=\frac{k_{\mathrm{e}} \tilde{\Gamma}^{2}}{\alpha_{0} \gamma^{2}} I_{p}$

where constant $\alpha_{0}$ is defined in Sect. 3.1.1.

$$
\begin{aligned}
I_{p} & =\int_{\gamma}^{\min \left(\gamma_{*}, \gamma_{\max }\right)} \mathrm{d} \tilde{\gamma} \tilde{\gamma}^{-p}\left[\frac{1}{\sqrt{r}}+\frac{c \tilde{\Gamma}}{2 \alpha_{0}}\left(\frac{1}{\gamma}-\frac{1}{\tilde{\gamma}}\right)\right]^{-4} \\
\gamma_{*} & =\frac{1}{\frac{1}{\gamma}+\frac{2 \alpha_{0}}{c \tilde{\Gamma}}\left(\frac{1}{\sqrt{r}}-\frac{1}{\sqrt{r_{0}}}\right)} .
\end{aligned}
$$

We define $A=\frac{c \tilde{\Gamma}}{2 \alpha_{0}}$. The integral for $p>2$ (as it is typically assumed) can be estimated in two regimes:

\section{Uncooled}

In this regime $\gamma<\gamma_{\mathrm{c}}$ and $\gamma_{*}<\gamma_{\max }$. Taking also into account that $\gamma_{*}>\gamma$ and $p>2$ Eq. (A.4) becomes:

$I_{p}^{\text {uncooled }}=-\left.\frac{2 \alpha_{0}}{3 c \tilde{\Gamma}} \tilde{\gamma}^{-p+2}\left[\frac{1}{\sqrt{r}}+\frac{c \tilde{\Gamma}}{2 \alpha_{0}}\left(\frac{1}{\gamma}-\frac{1}{\tilde{\gamma}}\right)\right]^{-3}\right|_{\gamma} ^{\gamma_{*}}$

or

$I_{p}^{\text {uncooled }} \approx \frac{2 \alpha_{0}}{3 c \tilde{\Gamma}} r^{3 / 2} \gamma^{-p+2}$

$N_{\text {uncooled }} \approx \frac{2 k_{\mathrm{e}} \tilde{\Gamma}}{3 c} r^{3 / 2} \gamma^{-p}$.

\section{Cooled}

This is the regime where the bulk of the electron population has already cooled and corresponds to the conditions $\gamma>\gamma_{\mathrm{b}}$ and $\gamma \gg \gamma_{\mathrm{c}}$ or equivalently $\frac{A \sqrt{r}}{\gamma} \ll 1$, as mentioned in Sect. 3.1.1. The integral $I_{\mathrm{p}}$ is then calculated:

$$
I_{\mathrm{p}}=-\frac{1}{6}\left[f_{0}(\gamma, r ; p)\left[f_{1}(\gamma, r ; p) f_{2}(\gamma, r ; p)+f_{3}(\gamma, r ; p)\right]\right]
$$

where

$$
\begin{aligned}
f_{0}= & \frac{\gamma^{5}}{(p-1)\left(A+\frac{\gamma}{\sqrt{r}}\right)^{5}} \\
f_{1}= & -\frac{1}{\gamma^{3}}\left(-\frac{A+\frac{\gamma}{\sqrt{r}}}{\gamma}\right)^{p}\left(-\frac{\sqrt{r}}{\gamma+A \sqrt{r}}\right)^{p-1} \\
f_{2}= & -(\gamma+A \sqrt{r})(p-1) \\
& \cdot\left(2 A^{2} r-A \gamma \sqrt{r}(p-8)+\gamma^{2}\left(18-8 p+p^{2}\right)\right) \\
& +\gamma^{3}\left(-24+26 p-9 p^{2}+p^{3}\right) . \\
& \cdot F\left(1, p-1 ; p ; 1-\frac{\gamma}{\gamma+A \sqrt{r}}\right) \\
f_{3}= & 6 \gamma_{\max }^{-p+1}\left(\frac{A}{\gamma}+\frac{1}{\sqrt{r}}\right) \\
& \cdot F\left(4, p-1 ; p ; \frac{A \gamma}{\gamma_{\max }\left(A+\frac{\gamma}{\sqrt{r}}\right)}\right)
\end{aligned}
$$

where $F(a, b ; c ; z)$ is the Gaussian hypergeometric function. The hypergeometric function has a power series representation. The three first terms of the series are:

$$
\begin{aligned}
F(a, b ; c ; z)=1 & +\frac{a b}{1 ! c} z \\
& +\frac{a(a+1) b(b+1)}{2 ! c(c+1)} z^{2}+\ldots
\end{aligned}
$$

The condition $\frac{A \sqrt{r}}{\gamma} \ll 1$ allows us to expand each term of Eq. (A.9) in terms of $\frac{A \sqrt{r}}{\gamma}$. We work only in zeroth order, in order to obtain the simplest expression for $I_{\mathrm{p}}$ and its dependance on $r, \gamma$ :

$\frac{\gamma^{5}}{(p-1)\left(A+\frac{\gamma}{\sqrt{r}}\right)^{5}} \approx \frac{r^{5 / 2}}{p-1}$

$$
(-1)^{2 p} \frac{1}{\gamma^{3}}\left(\frac{A+\frac{\gamma}{\sqrt{r}}}{\gamma}\right)^{p}\left(\frac{\sqrt{r}}{\gamma+A \sqrt{r}}\right)^{p-1} \approx \gamma^{-p-2} r^{-1 / 2}
$$

$6 \gamma_{\max }^{-p+1}\left(\frac{A}{\gamma}+\frac{1}{\sqrt{r}}\right) \approx \frac{6 \gamma_{\max }^{-p+1}}{\sqrt{r}}$

The term of equation (A.17) will not be taken into account later on. The argument of the hypergeometric function in Eq. (A.13) becomes

$\frac{A \gamma}{\gamma_{\max }\left(A+\frac{\gamma}{\sqrt{r}}\right)} \approx \frac{A \sqrt{r}}{\gamma_{\max }} \ll 1$.

Thus, we can approximate

$F\left(4, p-1 ; p ; \frac{A \gamma}{\gamma_{\max }\left(A+\frac{\gamma}{\sqrt{r}}\right)}\right) \approx 1$.

The hypergeometric function with argument $1-\frac{\gamma}{\gamma+A \sqrt{r}} \rightarrow 0$ can also be approximated by unity. With the above approximations, Eq. (A.9) reduces to:

$I_{\mathrm{p}}^{\text {cooled }} \approx \frac{1}{p-1} r^{2} \gamma^{-p+1}$.

Thus, the electron distribution in the cooled regime is given by the simple expression:

$N_{\text {cooled }} \approx \frac{k_{\mathrm{e}} \tilde{\Gamma}^{2}}{\alpha_{0}(p-1)} r^{2} \gamma^{-p-1}$.

\section{A.2. Magnetic field of the form $B(r)=B_{0} \frac{r_{0}}{r}$}

The general solution of Eq. (11) is

$N(\gamma, r)=\int_{-\infty}^{r} \mathrm{~d} \tilde{r} Q(\tilde{\gamma}, \tilde{r}) \frac{\tilde{\gamma}^{2}}{\gamma^{2}}$

or changing the integration variable

$N(\gamma, r)=\frac{1}{\alpha_{0} \gamma^{2}} \int_{\gamma}^{\infty} \mathrm{d} \tilde{\gamma} Q(\tilde{\gamma}, \tilde{r}) c \Gamma(\tilde{r}) \tilde{r}^{2}$ 
where constant $\alpha_{0}$ is defined in Sect. (3.1.2). Assuming that the blast wave is in the decelerating phase (Eq. (4)) the above equation becomes:

$N(\gamma, r)=\frac{k_{\mathrm{e}} \tilde{\Gamma}^{2}}{\alpha_{0} \gamma^{2}} I_{p}$

where

$I_{p}=\int_{\gamma}^{\min \left(\gamma_{*}, \gamma_{\max }\right)} \mathrm{d} \tilde{\gamma} \tilde{\gamma}^{-p}\left[\sqrt{r}-\frac{c \tilde{\Gamma}}{2 \alpha_{0}}\left(\frac{1}{\gamma}-\frac{1}{\tilde{\gamma}}\right)\right]^{2}$

$\gamma_{*}=\frac{1}{\frac{1}{\gamma}-\frac{2 \alpha_{0}}{c \tilde{\Gamma}}\left(\sqrt{r}-\sqrt{r_{0}}\right)}$.

If $p \neq 2$ the integral $I_{p}$ can only be estimated in two regimes:

\section{- Uncooled}

In this regime, $\gamma<\gamma_{\mathrm{c}}$ and $\gamma_{*}<\gamma_{\max }$. Equation (A.25) becomes

$I_{p}^{\text {uncooled }}=-\left.\frac{2 \alpha_{0}}{3 c \tilde{\Gamma}} \tilde{\gamma}^{-p+2}\left[\sqrt{r}-\frac{c \tilde{\Gamma}}{2 \alpha_{0}}\left(\frac{1}{\gamma}-\frac{1}{\tilde{\gamma}}\right)\right]^{3}\right|_{\gamma} ^{\gamma_{*}}$.

Taking into account that $\gamma_{*}>\gamma$ and $p>2$ (as it is typically assumed), inspection of (A.27) shows that:

$$
\begin{aligned}
I_{p}^{\text {uncooled }} & \approx \frac{2 \alpha_{0}}{3 c \tilde{\Gamma}} r^{3 / 2} \gamma^{-p+2} \\
N_{\text {uncooled }} & \approx \frac{2 k_{\mathrm{e}} \tilde{\Gamma}}{3 c} r^{3 / 2} \gamma^{-p} .
\end{aligned}
$$

\section{- Cooled}

This is the regime where the bulk of the electron population has already cooled and corresponds to the condition $\gamma>\gamma_{\mathrm{b}}$ or equivalently $\gamma_{*}>\gamma_{\max }$. When $\gamma \ll \gamma_{\max }$

$I_{p} \rightarrow I_{p}^{\text {cooled }}=\frac{\gamma^{-p+1}}{p-1}\left(\sqrt{r}-\frac{c \tilde{\Gamma}}{2 \alpha_{0} \gamma}\right)^{2}$.

Moreover the condition $\gamma_{*}>\gamma_{\max }$ leads to $\frac{c \tilde{\Gamma}}{2 \alpha_{0} \gamma}<\sqrt{r}$. However, we can safely neglect the term $\frac{c \tilde{\Gamma}}{2 \alpha_{0} \gamma}$, only if it is much smaller than the term $\sqrt{r}$. This condition, as discussed in Sect. 3.1.1, is the same as $\gamma \gg \gamma_{\mathrm{c}}$. Under this approximation:

$$
\begin{aligned}
I_{p}^{\text {cooled }} & \approx \frac{\gamma^{-p+1}}{p-1} r \\
N_{\text {cooled }} & \approx \frac{k_{\mathrm{e}} \tilde{\Gamma}^{2}}{\alpha_{0}(p-1)} r \gamma^{-p-1} .
\end{aligned}
$$

\section{A.3. Constant magnetic field}

The solution of Eq. (11) is:

$N(\gamma, r)=\int_{-\infty}^{r} \mathrm{~d} \tilde{r} Q(\tilde{\gamma}, \tilde{r}) \frac{\tilde{\gamma}^{2}}{\gamma^{2}}$

or changing the integration variable

$N(\gamma, r)=\frac{1}{\alpha_{0} \gamma^{2}} \int_{\gamma}^{\infty} \mathrm{d} \tilde{\gamma} Q(\tilde{\gamma}, \tilde{r}) c \Gamma(\tilde{r})$ where constant $\alpha_{0}$ is defined in Sect. (3.1.3). As described in the previous sections, the solution in terms of the integral $I_{p}$ is:

$N(\gamma, r)=\frac{k_{\mathrm{e}} \tilde{\Gamma}^{2}}{\alpha_{0} \gamma^{2}} I_{p}$

where in this case

$$
\begin{aligned}
I_{p} & =\int_{\gamma}^{\min \left(\gamma_{*}, \gamma_{\max }\right)} \mathrm{d} \tilde{\gamma} \tilde{\gamma}^{-p}\left[r^{5 / 2}-\frac{5 c \tilde{\Gamma}}{2 \alpha_{0}}\left(\frac{1}{\gamma}-\frac{1}{\tilde{\gamma}}\right)\right]^{-2 / 5} \\
\gamma_{*} & =\frac{1}{\frac{1}{\gamma}-\frac{2 \alpha_{0}}{5 c \tilde{\Gamma}}\left(r^{5 / 2}-r_{0}^{5 / 2}\right)}
\end{aligned}
$$

We outline next the points needed that will faciliate a comparison of our analytical results and those presented in (Dermer \& Chiang 1998, hereafter DC98). The expressions given by Eqs. (A.35), (A.36) coincide with these of Eqs. (33), (34) of DC98. This can be seen after taking into account the corresponding symbolism of our present work with the one used in DC98. The spatial coordinates $x, x_{0}$, used in DC98, and $r, r_{0}$, used in our work, are identical. In the case of a spherical blast wave

$A_{0}=4 \pi r_{0}^{2}$.

When the bulk of the electron distribution is considered to be relativistic then $\beta \approx 1$ and

$p=\beta \gamma \approx \gamma$.

The constant $\tilde{\Gamma}$ used throughout our paper is related to $\Gamma_{0}, x_{0}$ of DC98 by

$\tilde{\Gamma}=\Gamma_{0} x_{0}^{3 / 2}$.

Our constant $k_{\mathrm{e}}$ is related to the constant in the right hand side of Eq. (33) of DC98 by the relation

$k_{\mathrm{e}}=\frac{\xi_{\mathrm{e}} 4 \pi \rho_{0} c}{m_{\mathrm{e}} f}$,

where $\xi_{\mathrm{e}} \equiv \epsilon_{\mathrm{e}}$ and $\rho_{0}=m_{\mathrm{p}} n_{0}$. The relationship between the two integrals $I_{p}(\tau)$ in DC98 and $I_{p}(\gamma, r)$ in our paper is given by

$I_{p}(\tau)=\frac{r_{0}}{\alpha_{0}} I_{p}(\gamma, r)$

Moreover, the constants $v_{0}$ and $\omega$ which appear in Eq. (34) of DC98 can be expressed as

$v_{0} \equiv \alpha_{0}$

$\omega=\frac{5 \tilde{\Gamma} c}{2 r_{0}^{5 / 2}}$.

Also the variable $\tau$ of DC98 can be expressed in terms of the distance $r$ measured in the frame of the explosion by the relation

$\tau=\frac{2}{5 c \tilde{\Gamma}}\left(r^{5 / 2}-r_{0}^{5 / 2}\right)$.

Finally the exponent $u$ which appears in the integral $I_{p}(\tau)$ takes the value $-\frac{2}{5}$. The characteristic Lorentz factor $\gamma_{\mathrm{b}}$ which divides the analytic solution of Eq. (A.35) into two branches is given by:

$\gamma_{\mathrm{b}}=\frac{1}{\frac{1}{\gamma_{\max }}+\frac{2 \alpha_{0}}{5 c \tilde{\Gamma}}\left(r^{5 / 2}-r_{0}^{5 / 2}\right)}$. 
Taking into account $\gamma_{\max } \gg 1$ and $r \gg r_{0}$ Eq. (A.46) becomes:

$\gamma_{\mathrm{b}} \approx \frac{5 c \tilde{\Gamma}}{2 \alpha_{0}} r^{-5 / 2} \equiv \gamma_{\mathrm{c}}$

Therefore, in this case the two characteristic Lorentz factors of the distribution $\gamma_{\mathrm{c}}, \gamma_{\mathrm{b}}$ coincide. In order to obtain the asymptotic solutions presented in Sect. 3.1.3 one follows the procedure presented in the previous sections.

\section{References}

Blandford, R. D., \& McKee, C. F. 1976, Phys. Fluids, 19, 1130 Bloom, S. D., \& Marscher, A. P. 1996, ApJ, 461, 657

Blumenthal, G. R., \& Gould, R. J. 1970, Rev. Mod. Phys., 42, 237 Böttcher, M. 2007, Ap\&SS, 309, 95

Chiang, J., \& Dermer, C. D. 1999, ApJ, 512, 699

Coppi, P. S. 1992, MNRAS, 258, 657

Dermer, C. D., \& Chiang, J. 1998, New Astron., 3, 157
Fan, Y.-Z., Piran, T., Narayan, R., \& Wei, D.-M. 2008, MNRAS, 384, 1483 Inoue, S., \& Takahara, F. 1996, ApJ, 463, 555

Kardashev, N. S. 1962, Sov. Astron., 6, 317

Katarzyński, K., Ghisellini, G., Tavecchio, F., Maraschi, L., Fossati, G., \& Mastichiadis, A. 2005, A\&A, 433, 479

Katz, J. I., \& Piran, T. 1997, ApJ, 490, 772

Konopelko, A., Mastichiadis, A., Kirk, J., de Jager, O. C., \& Stecker, F. W. 2003, ApJ, 597, 851

Krawczynski, H., Coppi, P. S., \& Aharonian, F. 2002, MNRAS, 336, 721

Maraschi, L., Ghisellini, G., \& Celotti, A. 1992, ApJ, 397, L5

Mastichiadis, A. 2009, International Journal of Modern Physics D, in press

Mastichiadis, A., \& Kazanas, D. 2009, ApJ, 694, L54

Mastichiadis, A., \& Kirk, J. G. 1995, A\&A, 295, 613

Mastichiadis, A., \& Kirk, J. G. 1997, A\&A, 320, 19

Panaitescu, A., \& Kumar, P. 2000, ApJ, 543, 66

Panaitescu, A., \& Mészáros, P. 1998, ApJ, 492, 683

Piran, T. 2005, Rev. Mod. Phys., 76, 1143

Rees, M. J., \& Mészáros, P. 1992, MNRAS, 258, 41P

Sari, R., Piran, T., \& Narayan, R. 1998, ApJ, 497, L17

Vlahakis, N., \& Königl, A. 2003, Ap\&SS, 287, 249

Waxman, E. 1997, ApJ, 485, L5 